九州大学学術情報リポジトリ

Kyushu University Institutional Repository

\title{
Hypothetic Incomplete Pair Disappearances of Comp lex Numbers Forming ' $0=(-1)+1$ ' in the Application to Complex Representation of Some Aspects of Ruminant Agriculture
}

Shimojo, Masataka

Laboratory of Animal Feed Science, Division of Animal Science, Department of Animal and Marine Bioresource Sciences, Faculty of Agriculture, Kyushu University

Asano, Yoki

Research Fellow, Faculty of Agriculture, Kyushu University

Ikeda, Kentarou

Research Fellow, Faculty of Agriculture, Kyushu University

Ishiwaka, Reiko

Research Fellow, Faculty of Agriculture, Kyushu University

他

https://doi.org/10.5109/4565

出版情報: 九州大学大学院農学研究院紀要. 49 (1)，pp.61-67，2004-02-01. Faculty of Agriculture， Kyushu University

バージョン :

権利関係 : 


\title{
Hypothetic Incomplete Pair Disappearances of Complex Numbers Forming ' $0=(-1)+1$ ' in the Application to Complex Representation of Some Aspects of Ruminant Agriculture
}

\author{
Masataka SHIMOJO*, Kentarou IKEDA ${ }^{1}$, Yoki ASANO ${ }^{1}$, Reiko ISHIWAKA ${ }^{1}$, \\ Tao SHAO ${ }^{2}$, Hiroyuki SATO ${ }^{3}$, Manabu TOBISA, Yutaka NAKANO 4 , \\ Noriko OHBA $^{5}$, Yasukatsu YANO ${ }^{6}$ and Yasuhisa MASUDA
}

\author{
Laboratory of Animal Feed Science, Division of Animal Science, Department of \\ Animal and Marine Bioresource Sciences, Faculty of Agriculture, \\ Kyushu University, Fukuoka 812-8581, Japan \\ (Received October 31, 2003 and accepted November 14, 2003)
}

\begin{abstract}
The present study was conducted to investigate the complex representation of ' $0=(-1)+1$ ' in order to leave some complex numbers for the complex representation of some aspects of ruminant agriculture. The results obtained were as follows: (i) There were two systems for the complex representation of ' $0=(-1)+1$ '; namely four-component system and eight-component system. (ii) The four-component system using four complex numbers to describe each of '-1' and ' 1 ' left real numbers or imaginary numbers through a hypothetic breakdown of bonding that connected four components, suggesting unavailability for the complex representation. (iii) The eight-component system, that was constructed by eight complex numbers forming ' 1 ' and giving a minus sign to one of the eight components to form ' -1 ', left complex numbers through the hypothetic breakdown of bonding, suggesting availability for the complex representation of some micro- and macro-structures in ruminant agriculture.
\end{abstract}

\section{INTRODUCTION}

It was suggested in our previous reports (Shimojo et al., 2003a, b, c, d, e) that the complex representation of a real number ' 1 ' gave a kind of tool to simple descriptions of some micro- and macro-structures in ruminant agriculture. In these reports, ' 1 ' was described as the product of eight components (four sets of a complex number and its opposite complex number); Euler's formula, its conjugate complex and three phase shifts for each of the two. In addition the following two hypothetic procedures were introduced in order to examine the behavior of the eight components (Shimojo et al., 2003e). (1) The borrowing of ' 1 ' from the seeming nothing was followed by returning it to the

1 Research Fellow, Faculty of Agriculture, Kyushu University

2 Visiting Research Scientist from Lanzhou Institute of Animal Science and Veterinary Medicine of CAAS, Lanzhou Gansu Province of the People's Republic of China

${ }^{3}$ Laboratory of Animal Feed Science, Division of Animal Science, Department of Animal and Marine Bioresource Sciences, Graduate School of Bioresource and Bioenvironmental Sciences, Kyushu University

4 Kyushu University Farm, Fukuoka 811-2307

${ }^{5}$ Research Student, School of Agriculture, Kyushu University

${ }^{6}$ Former Technical Specialist

* Corresponding author (E-mail: mshimojo@agr.kyushu-u.ac.jp) 
creditor, namely ' $0 \rightarrow 1 \rightarrow 0$ '. (2) This was achieved by a hypothetic breakdown of the form of multiplication connecting the eight complex numbers forming ' 1 ', which resulted in ' 0 ' through pair appearances and pair disappearances of them.

Moreover, another way using the equality ' $0=1+(-1)^{\prime}$ ' may be taken up, where there is the complex representation of ' -1 ' as well as ' 1 '. Since ' -1 ' results from giving a minus sign to one of the eight complex numbers forming ' 1 ', the aspect of pair disappearances in ' -1 ' will be different from that in ' 1 ' when there is an application of hypothetic breakdown of bonding (multiplication) in the eight components. This might be expected to show a kind of incomplete pair disappearances in '-1', leaving some complex numbers.

The present study was designed to investigate hypothetic incomplete pair disappearances of complex numbers forming ' $0=(-1)+1$ ' for the complex representation of some aspects of ruminant agriculture.

\section{COMPLEX REPRESENTATION OF ' -1 ' AND ' 1 '}

\section{Complex representation of ' -1 ' and ' 1 '}

The complex representation of ' -1 ' and ' 1 ' is given by the procedure suggested by Shimojo et al. (2003a, b, d, e). Thus,

$$
\begin{aligned}
& -1=\boldsymbol{i} \cdot \boldsymbol{i}=(\cos \gamma+\boldsymbol{i} \sin \gamma) \cdot(\sin \gamma+\boldsymbol{i} \cos \gamma) \cdot(-\cos \gamma-\boldsymbol{i} \sin \gamma) \cdot(-\sin \gamma-\boldsymbol{i} \cos \gamma) \\
& -1=(-\boldsymbol{i}) \cdot(-\boldsymbol{i})=(-\sin \gamma+\boldsymbol{i} \cos \gamma) \cdot(-\cos \gamma+\boldsymbol{i} \sin \gamma) \cdot(\sin \gamma-\boldsymbol{i} \cos \gamma) \cdot(\cos \gamma-\boldsymbol{i} \sin \gamma) \\
& 1=\boldsymbol{i} \cdot(-\boldsymbol{i})=(\cos \gamma+\boldsymbol{i} \sin \gamma) \cdot(\sin \gamma+\boldsymbol{i} \cos \gamma) \cdot(-\sin \gamma+\boldsymbol{i} \cos \gamma) \cdot(-\cos \gamma+\boldsymbol{i} \sin \gamma) \\
& 1=(-\boldsymbol{i}) \cdot \boldsymbol{i}=(-\sin \gamma+\boldsymbol{i} \cos \gamma) \cdot(-\cos \gamma+\boldsymbol{i} \sin \gamma) \cdot(-\cos \gamma-\boldsymbol{i} \sin \gamma) \cdot(-\sin \gamma-\boldsymbol{i} \cos \gamma) \\
& 1=\boldsymbol{i} \cdot(-\boldsymbol{i})=(-\cos \gamma-\boldsymbol{i} \sin \gamma) \cdot(-\sin \gamma-\boldsymbol{i} \cos \gamma) \cdot(\sin \gamma-\boldsymbol{i} \cos \gamma) \cdot(\cos \gamma-\boldsymbol{i} \sin \gamma) \\
& 1=(-\boldsymbol{i}) \cdot \boldsymbol{i}=(\sin \gamma-\boldsymbol{i} \cos \gamma) \cdot(\cos \gamma-\boldsymbol{i} \sin \gamma) \cdot(\cos \gamma+\boldsymbol{i} \sin \gamma) \cdot(\sin \gamma+\boldsymbol{i} \cos \gamma)
\end{aligned}
$$

There are two complex descriptions of '-1' [(1) and (2)], where each description is composed of four components, namely two sets of a complex number and its opposite complex number. The product of (1) and (2) gives the eight components that are composed of four sets of a complex number and its opposite complex number, and there appears ' 1 ' as a result of ' $(-1) \cdot(-1)$ '. There are four complex descriptions [(3), (4), (5) and (6)] for ' 1 ', where each of these has four components that are not composed of two sets of a complex number and its opposite complex number. The product of (3) and (5) and that of (4) and (6), which give ' 1 ', are required in order to have the eight components composed of four sets of a complex number and its opposite complex number. The other combinations $[(3) \cdot(3),(3) \cdot(4),(3) \cdot(6),(4) \cdot(4),(4) \cdot(5),(5) \cdot(5),(5) \cdot(6)$ and $(6) \cdot(6)]$ do not satisfy the required condition. This is why the complex representation of ' 1 ' that is given by $(1) \cdot(2),(3) \cdot(5)$ and $(4) \cdot(6)$ with a distribution over the four quadrants of complex plane is used in order to give simple descriptions of some aspects of ruminant agriculture (Shimojo et al., 2003a, b, c, d, e). It is also shown by Shimojo et al. (2003e) that the borrowing of ' 1 ' from the seeming nothing is followed by returning it to the creditor, where there is a hypothetic breakdown of bonding of eight components forming ' 1 ', resulting in ' 0 '.

Since the present study is based on the equality ' $0=(-1)+1$ ', the next section will take up an application of hypothetic procedures to the complex representation of ' $0=$ 
$(-1)+1$

\section{Applying the hypothetic breakdown of bonding to the complex representation} of ' $0=(-1)+1$ '

In this section, two systems are taken up for the complex representation of ' $0=$ $(-1)+1$ ', namely the system using four components and that using eight components for describing each of ' -1 ' and ' 1 '.

\section{Four-component system}

There are eight descriptions that are given by the addition of one of $(1) \sim(2)$ and one of (3) (6). The addition of (1) and (3) and that of (2) and (6) are considered enough to show a broad outline of four-component system.

Thus, the addition of (1) and (3) is given by

$$
\begin{aligned}
0= & (-1)+1 \\
= & \{(\cos \gamma+\boldsymbol{i} \sin \gamma) \cdot(\sin \gamma+\boldsymbol{i} \cos \gamma) \cdot(-\cos \gamma-\boldsymbol{i} \sin \gamma) \cdot(-\sin \gamma-\boldsymbol{i} \cos \gamma)\} \\
& +\{(\cos \gamma+\boldsymbol{i} \sin \gamma) \cdot(\sin \gamma+\boldsymbol{i} \cos \gamma) \cdot(-\sin \gamma+\boldsymbol{i} \cos \gamma) \cdot(-\cos \gamma+\boldsymbol{i} \sin \gamma)\} .
\end{aligned}
$$

If the hypothetic breakdown of bonding is applied to (7), then

$$
\begin{aligned}
& \{(\cos \gamma+\boldsymbol{i} \sin \gamma)+(\sin \gamma+\boldsymbol{i} \cos \gamma)+(-\cos \gamma-\boldsymbol{i} \sin \gamma)+(-\sin \gamma-\boldsymbol{i} \cos \gamma)\} \\
& +\{(\cos \gamma+\boldsymbol{i} \sin \gamma)+(\sin \gamma+\boldsymbol{i} \cos \gamma)+(-\sin \gamma+\boldsymbol{i} \cos \gamma)+(-\cos \gamma+\boldsymbol{i} \sin \gamma)\} \\
& =2 \boldsymbol{i}(\cos \gamma+\sin \gamma) .
\end{aligned}
$$

The addition of (2) and (6) is given by

$$
\begin{aligned}
0= & (-1)+1 \\
= & \{(-\sin \gamma+\boldsymbol{i} \cos \gamma) \cdot(-\cos \gamma+\boldsymbol{i} \sin \gamma) \cdot(\sin \gamma-\boldsymbol{i} \cos \gamma) \cdot(\cos \gamma-\boldsymbol{i} \sin \gamma)\} \\
& +\{(\sin \gamma-\boldsymbol{i} \cos \gamma) \cdot(\cos \gamma-\boldsymbol{i} \sin \gamma) \cdot(\cos \gamma+\boldsymbol{i} \sin \gamma) \cdot(\sin \gamma+\boldsymbol{i} \cos \gamma)\} .
\end{aligned}
$$

Applying the hypothetic breakdown of bonding to (8) gives

$$
\begin{aligned}
& \{(-\sin \gamma+\boldsymbol{i} \cos \gamma)+(-\cos \gamma+\boldsymbol{i} \sin \gamma)+(\sin \gamma-\boldsymbol{i} \cos \gamma)+(\cos \gamma-\boldsymbol{i} \sin \gamma)\} \\
& +\{(\sin \gamma-\boldsymbol{i} \cos \gamma)+(\cos \gamma-\boldsymbol{i} \sin \gamma)+(\cos \gamma+\boldsymbol{i} \sin \gamma)+(\sin \gamma+\boldsymbol{i} \cos \gamma)\} \\
& =2(\cos \gamma+\sin \gamma) .
\end{aligned}
$$

The equations $(7) \sim(10)$ show that if ' $0=(-1)+1$ ', that is composed of four complex numbers for each of ' -1 ' and ' 1 ', is given the hypothetic breakdown of bonding, then the imaginary number $[2 \boldsymbol{i}(\cos \gamma+\sin \gamma)]$ or the real number $[2(\cos \gamma+\sin \gamma)]$ remains and the others vanish through pair disappearances. The components that are left come from ' 1 ', because there are complete pair disappearances in the case of ' -1 '. This might give an impression that there is an emergence of $2 \boldsymbol{i}(\cos \gamma+\sin \gamma)$ or $2(\cos \gamma+\sin \gamma)$ from the seeming nothing. However, this does not apply to complex representation of some aspects of ruminant agriculture, because they are described using complex numbers (Shimojo et al., 2003a, b, c, d, e). It goes without saying, however, that no complex num- 
ber is left when the hypothetic breakdown of bonding does not occur, where ' $0 \rightarrow(-1)+1$ ' is followed by ' $(-1)+1 \rightarrow 0$ ', a kind of fluctuation around ' 0 '

\section{Eight-component system}

The complex representation of ' 1 ' using eight components is given by

$$
\begin{array}{r}
1=(\cos \gamma+\boldsymbol{i} \sin \gamma) \cdot(\sin \gamma+\boldsymbol{i} \cos \gamma) \cdot(-\sin \gamma+\boldsymbol{i} \cos \gamma) \cdot(-\cos \gamma+\boldsymbol{i} \sin \gamma) \\
\cdot(-\cos \gamma-\boldsymbol{i} \sin \gamma) \cdot(-\sin \gamma-\boldsymbol{i} \cos \gamma) \cdot(\sin \gamma-\boldsymbol{i} \cos \gamma) \cdot(\cos \gamma-\boldsymbol{i} \sin \gamma)
\end{array}
$$

The product of one of $(1) \sim(2)$ and one of (3) (6) gives ' -1 ', but these eight combinations give real numbers or imaginary numbers when the hypothetic breakdown of bonding is applied. Therefore, other ways should be taken up. An easy way to describe ' -1 ' using eight components is to give a minus sign to one of the components of ' 1 '. Thus, the following is given as an example,

$$
\begin{aligned}
-1= & (\cos \gamma+\boldsymbol{i} \sin \gamma) \cdot(\sin \gamma+\boldsymbol{i} \cos \gamma) \cdot(-\sin \gamma+\boldsymbol{i} \cos \gamma) \cdot(-\cos \gamma+\boldsymbol{i} \sin \gamma) \\
& \cdot\{-(-\cos \gamma-\boldsymbol{i} \sin \gamma)\} \cdot(-\sin \gamma-\boldsymbol{i} \cos \gamma) \cdot(\sin \gamma-\boldsymbol{i} \cos \gamma) \cdot(\cos \gamma-\boldsymbol{i} \sin \gamma)
\end{aligned}
$$

The addition of (11) and (12) is considered enough to show a broad outline of eight-component system. Thus,

$$
\begin{aligned}
0= & (-1)+1 \\
= & {[(\cos \gamma+\boldsymbol{i} \sin \gamma) \cdot(\sin \gamma+\boldsymbol{i} \cos \gamma) \cdot(-\sin \gamma+\boldsymbol{i} \cos \gamma) \cdot(-\cos \gamma+\boldsymbol{i} \sin \gamma)} \\
& \cdot(-(-\cos \gamma-\boldsymbol{i} \sin \gamma) \cdot(-\sin \gamma-\boldsymbol{i} \cos \gamma) \cdot(\sin \gamma-\boldsymbol{i} \cos \gamma) \cdot(\cos \gamma-\boldsymbol{i} \sin \gamma)] \\
+ & {[(\cos \gamma+\boldsymbol{i} \sin \gamma) \cdot(\sin \gamma+\boldsymbol{i} \cos \gamma) \cdot(-\sin \gamma+\boldsymbol{i} \cos \gamma) \cdot(-\cos \gamma+\boldsymbol{i} \sin \gamma)} \\
& \cdot(-\cos \gamma-\boldsymbol{i} \sin \gamma) \cdot(-\sin \gamma-\boldsymbol{i} \cos \gamma) \cdot(\sin \gamma-\boldsymbol{i} \cos \gamma) \cdot(\cos \gamma-\boldsymbol{i} \sin \gamma)] .
\end{aligned}
$$

If the hypothetic breakdown of bonding is applied to (13), then

$$
\begin{aligned}
& {[(\cos \gamma+\boldsymbol{i} \sin \gamma)+(\sin \gamma+\boldsymbol{i} \cos \gamma)+(-\sin \gamma+\boldsymbol{i} \cos \gamma)+(-\cos \gamma+\boldsymbol{i} \sin \gamma)} \\
& \quad-(-\cos \gamma-\boldsymbol{i} \sin \gamma)+(-\sin \gamma-\boldsymbol{i} \cos \gamma)+(\sin \gamma-\boldsymbol{i} \cos \gamma)+(\cos \gamma-\boldsymbol{i} \sin \gamma)] \\
& +[(\cos \gamma+\boldsymbol{i} \sin \gamma)+(\sin \gamma+\boldsymbol{i} \cos \gamma)+(-\sin \gamma+\boldsymbol{i} \cos \gamma)+(-\cos \gamma+\boldsymbol{i} \sin \gamma) \\
& \quad+(-\cos \gamma-\boldsymbol{i} \sin \gamma)+(-\sin \gamma-\boldsymbol{i} \cos \gamma)+(\sin \gamma-\boldsymbol{i} \cos \gamma)+(\cos \gamma-\boldsymbol{i} \sin \gamma)] \\
& =2(\cos \gamma+\boldsymbol{i} \sin \gamma) .
\end{aligned}
$$

The equations (11) (14) show that if ' $0=(-1)+1$ ', that is composed of eight complex numbers for each of '-1' and ' 1 ', is given the hypothetic breakdown of bonding, then the complex number $[2(\cos \gamma+i \sin \gamma)]$ remains and the others vanish through pair disappearances. The component that is left comes from ' -1 ', because there are complete pair disappearances in the case of ' 1 '. This might give an impression that there is an emergence of $2(\cos \gamma+\boldsymbol{i} \sin \gamma)$ from the seeming nothing. Broadly speaking at the risk of making mistakes, there might be a kind of generative power in ' 0 ' when it is expressed using complex numbers. The manipulation of giving a minus sign to one of the components in eight-component system leads to the other seven complex numbers when ' $0=$ $(-1)+1$ ' is used. For example, giving a minus sign to $\cos \gamma+\boldsymbol{i} \sin \gamma$ leads to an emer- 
gence of $-2(\cos \gamma+\boldsymbol{i} \sin \gamma)$ that is an opposite complex number to $2(\cos \gamma+\boldsymbol{i} \sin \gamma)$. This suggests that the eight-component system forming ' $0=(-1)+1$ ' is available for the complex representation of some aspects of ruminant agriculture. However, when the hypothetic breakdown of bonding does not occur, no complex number is left and there is a kind of fluctuation around ' 0 ' [' $0 \rightarrow\{(-1)+1\} \rightarrow 0$ '] that is shown by pair appearance and pair disappearance of ' -1 ' and ' 1 '.

\section{Other properties suggested from the complex representation of ' $0=(-1)+1$ '}

The number of components forming ' 1 ' will be expanded from 8 to $8 n(n=2,3,4, \cdots$ $\cdots$ ) due to the equality ' $1=1^{n '}$ (Shimojo et al., 2003e), and in addition, ' -1 ' results from giving a minus sign to one of the $8 n$ components forming ' 1 '. Thus,

$$
\begin{aligned}
0= & (-1)+1 \\
= & {\left[\left\{\left(\cos \gamma_{1}+\boldsymbol{i} \sin \gamma_{1}\right) \cdot\left(\sin \gamma_{1}+\boldsymbol{i} \cos \gamma_{1}\right) \cdot\left(-\sin \gamma_{1}+\boldsymbol{i} \cos \gamma_{1}\right) \cdot\left(-\cos \gamma_{1}+\boldsymbol{i} \sin \gamma_{1}\right)\right.\right.} \\
& \left.\cdot\left(-\left(-\cos \gamma_{1}-\boldsymbol{i} \sin \gamma_{1}\right)\right) \cdot\left(-\sin \gamma_{1}-\boldsymbol{i} \cos \gamma_{1}\right) \cdot\left(\sin \gamma_{1}-\boldsymbol{i} \cos \gamma_{1}\right) \cdot\left(\cos \gamma_{1}-\boldsymbol{i} \sin \gamma_{1}\right)\right\} \\
& \cdot\left\{\prod _ { k = 2 } ^ { n } \left\{\left(\cos \gamma_{k}+\boldsymbol{i} \sin \gamma_{k}\right) \cdot\left(\sin \gamma_{k}+\boldsymbol{i} \cos \gamma_{k}\right) \cdot\left(-\sin \gamma_{k}+\boldsymbol{i} \cos \gamma_{k}\right) \cdot\left(-\cos \gamma_{k}+\boldsymbol{i} \sin \gamma_{k}\right)\right.\right. \\
& \left.\left.\left.\cdot\left(-\cos \gamma_{k}-\boldsymbol{i} \sin \gamma_{k}\right) \cdot\left(-\sin \gamma_{k}-\boldsymbol{i} \cos \gamma_{k}\right) \cdot\left(\sin \gamma_{k}-\boldsymbol{i} \cos \gamma_{k}\right) \cdot\left(\cos \gamma_{k}-\boldsymbol{i} \sin \gamma_{k}\right)\right\}\right\}\right] \\
+ & \prod_{k=1}^{n}\left\{\left(\cos \gamma_{k}+\boldsymbol{i} \sin \gamma_{k}\right) \cdot\left(\sin \gamma_{k}+\boldsymbol{i} \cos \gamma_{k}\right) \cdot\left(-\sin \gamma_{k}+\boldsymbol{i} \cos \gamma_{k}\right) \cdot\left(-\cos \gamma_{k}+\boldsymbol{i} \sin \gamma_{k}\right)\right. \\
& \left.\cdot\left(-\cos \gamma_{k}-\boldsymbol{i} \sin \gamma_{k}\right) \cdot\left(-\sin \gamma_{k}-\boldsymbol{i} \cos \gamma_{k}\right) \cdot\left(\sin \gamma_{k}-\boldsymbol{i} \cos \gamma_{k}\right) \cdot\left(\cos \gamma_{k}-\boldsymbol{i} \sin \gamma_{k}\right)\right\},(15)
\end{aligned}
$$

where $0<\gamma_{1}<\cdots<\gamma_{k}<\cdots \gamma_{n}<\pi / 2$.

The application of hypothetic breakdown of bonding to (15) gives

$$
\begin{aligned}
& {\left[\left\{\left(\cos \gamma_{1}+\boldsymbol{i} \sin \gamma_{1}\right)+\left(\sin \gamma_{1}+\boldsymbol{i} \cos \gamma_{1}\right)+\left(-\sin \gamma_{1}+\boldsymbol{i} \cos \gamma_{1}\right)+\left(-\cos \gamma_{1}+\boldsymbol{i} \sin \gamma_{1}\right)\right.\right.} \\
& \left.\quad-\left(-\cos \gamma_{1}-\boldsymbol{i} \sin \gamma_{1}\right)+\left(-\sin \gamma_{1}-\boldsymbol{i} \cos \gamma_{1}\right)+\left(\sin \gamma_{1}-\boldsymbol{i} \cos \gamma_{1}\right)+\left(\cos \gamma_{1}-\boldsymbol{i} \sin \gamma_{1}\right)\right\} \\
& +\left\{\sum _ { k = 2 } ^ { n } \left\{\left(\cos \gamma_{k}+\boldsymbol{i} \sin \gamma_{k}\right)+\left(\sin \gamma_{k}+\boldsymbol{i} \cos \gamma_{k}\right)+\left(-\sin \gamma_{k}+\boldsymbol{i} \cos \gamma_{k}\right)+\left(-\cos \gamma_{k}+\boldsymbol{i} \sin \gamma_{k}\right)\right.\right. \\
& \left.\left.\left.\quad+\left(-\cos \gamma_{k}-\boldsymbol{i} \sin \gamma_{k}\right)+\left(-\sin \gamma_{k}-\boldsymbol{i} \cos \gamma_{k}\right)+\left(\sin \gamma_{k}-\boldsymbol{i} \cos \gamma_{k}\right)+\left(\cos \gamma_{k}-\boldsymbol{i} \sin \gamma_{k}\right)\right\}\right\}\right] \\
& +\sum_{k=1}^{n}\left\{\left(\cos \gamma_{k}+\boldsymbol{i} \sin \gamma_{k}\right)+\left(\sin \gamma_{k}+\boldsymbol{i} \cos \gamma_{k}\right)+\left(-\sin \gamma_{k}+\boldsymbol{i} \cos \gamma_{k}\right)+\left(-\cos \gamma_{k}+\boldsymbol{i} \sin \gamma_{k}\right)\right. \\
& \left.\quad+\left(-\cos \gamma_{k}-\boldsymbol{i} \sin \gamma_{k}\right)+\left(-\sin \gamma_{k}-\boldsymbol{i} \cos \gamma_{k}\right)+\left(\sin \gamma_{k}-\boldsymbol{i} \cos \gamma_{k}\right)+\left(\cos \gamma_{k}-\boldsymbol{i} \sin \gamma_{k}\right)\right\} \\
& =2(\cos \gamma+\boldsymbol{i} \sin \gamma) .
\end{aligned}
$$

In this expanded case, the complex number $[2(\cos \gamma+\boldsymbol{i} \sin \gamma)]$ is left by the pair disappearance of $16 n-2$ complex numbers after the hypothetic breakdown of bonding. If the size of $n$ is extremely large, then the complex number that is left [for example, $2(\cos \gamma+$ $\boldsymbol{i} \sin \gamma)$ or $-2(\cos \gamma+\boldsymbol{i} \sin \gamma)]$ is a result of minute differences in number between complex numbers and their opposite complex numbers forming ' $0=(-1)+1$ '.

As shown in (15) and (16), there is a large variety of complex numbers due to many phase shifts in the complex representation of ' $0=(-1)+1$ ', where each complex number has a duplicate except one of the components forming ' -1 '. Enlarging ' $0=(-1)+1$ ' $m$ times $(m=2,3,4, \cdots \cdots)$, ' $m \cdot 0=m \cdot(-1)+m \cdot 1$ ', increases the number from 2 to $2 m$ for each complex number except one, producing many duplicates. Broadly speaking at the 
risk of making mistakes, if enlarging $m$ times might be accompanied by a hypothetic increase in size, then the size of $m \cdot(16 n)$ components is $m$ times as large as that of $16 n$ components. However, the density of complex numbers is $16 n$ for both ' $(-1)+1$ ' and ' $m$. $(-1)+m \cdot 1$ ', suggesting the same density of components for all sizes. In contrast, when ' 1 ' is replaced by ' $1{ }^{n}$ ', comparisons between (13) and (15) and between (14) and (16) might lead to an increase from 16 to $16 n$ ( $n$ times) in the density of complex numbers, though its size might not change due to ' $1=\left(1^{n}\right)$ '. These hypotheses require more investigations in future studies.

\section{Implications and Problems}

(1) When ' 0 ' is expressed as ' $0=(-1)+1$ ', ' 0 ' might not be a state of nothing but might be a state composed of 16 complex numbers 8 complex numbers for '-1' and 8 complex numbers for ' 1 ']. The complex representation of '-1' results from giving a minus sign to one of the eight complex numbers forming ' 1 '.

(2) The complex numbers forming ' $0=(-1)+1$ ' are composed of Euler's formula, its conjugate complex and their phase shifts of $\pi / 2$ and $-\pi / 2$ (Shimojo et al., 2003a, b, d, e). Each of the complex numbers forms a point, a string and a circle with changes in $\gamma$ on the complex plane, and in addition, forms a spiral in the stereographic representation (Yoshida, 2000; Shimojo et al., 2003d, e).

(3) There is an introduction, into the complex representation of ' $0=(-1)+1$ ', of hypothetic breakdown of bonding that connects complex numbers forming each of ' -1 ' and ' 1 '.

(4) No complex number is left when the breakdown of bonding does not occur, resulting in ' $0 \rightarrow\{(-1)+1\} \rightarrow 0$ ', a kind of fluctuation around ' 0 '.

(5) If there is a breakdown of bonding, then the complex number is left through incomplete pair disappearances in '-1', a phenomenon that is different from complete pair disappearances in ' 1 '. This might give an impression that the complex number emerges from the seeming nothing, suggesting a kind of generative power in ' 0 ' when expressed using complex numbers.

(6) The events (1) (5) suggest one of the reasons why ' -1 ' and its complex representation are required in order to leave some complex numbers for the complex representation of micro- and macro-structures in ruminant agriculture.

(7) However, these concepts remain to be examined in further studies.

\section{REFERENCES}

Shimojo, M., K. Ikeda, Y. Asano, R. Ishiwaka, T. Shao, H. Sato, M. Tobisa, Y. Nakano, N. Ohba, Y. Yano and Y. Masuda 2003a A symbolic representation of field-forage-ruminant relationships using polar form on the complex plane. J. Fac. Agr., Kyushu Univ., 47: 359-366

Shimojo, M., Y. Asano, K. Ikeda, R. Ishiwaka, T. Shao, H. Sato, M. Tobisa, Y. Nakano, N. Ohba, Y. Yano and Y. Masuda 2003b Complex representation of field-forage-ruminant relationships using symmetric properties of Euler's formula. J. Fac. Agr., Kyushu Univ., 47: 367-372

Shimojo, M., K. Ikeda, Y. Asano, R. Ishiwaka, T. Shao, H. Sato, M. Tobisa, Y. Nakano, N. Ohba, Y. Yano and Y. Masuda 2003c Exponential functions with base $e$ in growth analysis and deriving them from rotations of axes of time described using Euler's formula. J. Fac. Agr., Kyushu Univ., 48: 65-69

Shimojo, M., Y. Asano, K. Ikeda, R. Ishiwaka, T. Shao, H. Sato, M. Tobisa; Y. Nakano, N. Ohba, Y. Yano and Y. Masuda 2003d A stereographic representation of Euler's formula to show spirals and topological similarities to micro-structures in ruminants and forages. J. Fac. Agr., Kyushu Univ., 48: 71-75 
Shimojo, M., K. Ikeda, Y. Asano, R. Ishiwaka, T. Shao, H. Sato, M. Tobisa, Y. Nakano, N. Ohba, Y. Yano and Y. Masuda 2003e Philosophical properties of Euler's formula in its application to symbolic representation of some aspects of ruminant agriculture. J. Fac. Agr., Kyushu Univ., 48: 77-83

Yoshida, T. 2000 Emotion for imaginary number. Tokai University Press, Tokyo. (written in Japanese) 